\section{RMD Open}

Rheumatic \&

Musculoskeletal Diseases
To cite: Ukichi T, Yoshida K, Matsushima S, et al. MRI of skeletal muscles in patients with idiopathic inflammatory myopathies: characteristic findings and diagnostic performance in dermatomyositis. RMD Open 2019;5:e000850. doi:10.1136/ rmdopen-2018-000850

- Prepublication history and additional material for this paper are available online. To view these files, please visit the journal online (http://dx.doi. org/10.1136/rmdopen-2018000850).

This manuscript is based on work previously presented and published as a conference abstract at the 2017 American College of Rheumatology (ACR) and Association of Rheumatology Health Professionals (ARHP) Annual Meeting: Ukichi T, Yoshida K, Matsushima S, Kawakami G, Noda K, Furuya K, Kurosaka D. MRI of skeletal muscles in patients with dermatomyositis and polymyositis: novel and distinctive characteristic finding [(abstract]). Arthritis Rheumatol. 2017;69 (Suppl 10).

Received 2 November 2018 Revised 15 February 2019 Accepted 5 March 2019

Check for updates

(c) Author(s) (or their employer(s)) 2019. Re-use permitted under CC BY-NC. No commercial re-use. See rights and permissions. Published by BMJ.

For numbered affiliations see end of article.

Correspondence to Dr Ken Yoshida;

k.yoshida@jikei.ac.jp

\title{
MRI of skeletal muscles in patients with idiopathic inflammatory myopathies: characteristic findings and diagnostic performance in dermatomyositis
}

\author{
Taro Ukichi, ${ }^{1}$ Ken Yoshida, ${ }^{1}$ Satoshi Matsushima, ${ }^{2}$ Go Kawakami, ${ }^{2}$ \\ Kentaro Noda, ${ }^{1}$ Kazuhiro Furuya, ${ }^{1}$ Daitaro Kurosaka ${ }^{1}$
}

\section{ABSTRACT}

Objective To define the characteristic findings on MRI of skeletal muscles in patients with dermatomyositis (DM) relative to those in patients with other idiopathic inflammatory myopathies (IIMs) and to assess their diagnostic performance in DM.

Methods Thirty-six patients with DM, 17 patients with amyopathic DM, 19 patients with polymyositis and 16 patients with non-IIM classified by the 2017 European League Against Rheumatism/American College of Rheumatology criteria were included in this study. The following MRI findings (short-tau inversion recovery [STIR] and gadolinium-enhanced fat-suppressed T1-weighted imaging [Gd-T1WI]) for proximal limb muscles were compared between the disease groups and between myositis-specific autoantibodies/myositis-associated autoantibodies (MSAs/MAAs)-positive and MSAs/MAAsnegative groups: structures with high signal intensity (HSI) (subcutaneous, fascia, muscle); distributions of HSI areas in muscle (diffuse, patchy, peripheral) and patterns of $\mathrm{HSI}$ in muscle (honeycomb, foggy, strong HSI). Univariate, multivariate and receiver-operating characteristic [ROC] analyses were performed to assess the diagnostic performance of MRI in DM.

Results The characteristic MRI findings in patients with DM were subcutaneous HSI, fascial HSI, peripheral distribution and honeycomb pattern. The MRI findings in the MSAs/ MAAs-positive group included more frequent fascial HSI but less frequent foggy pattern compared with the MSAs/MAAsnegative group. Likelihood of DM score $\geq 3$ (obtained by counting the number of characteristic MRI findings in patients with DM) showed good diagnostic performance in DM (STIR: sensitivity $72.2 \%$, specificity $88.5 \%$, area under ROC curve [AUC] 84.9\%; Gd-T1Wl: sensitivity $81.2 \%$, specificity $91.5 \%$, AUC 89.9\%)

Conclusion The characteristic MRI findings of skeletal muscles can predict patients with DM as well as patients with MSAs/MAAs.

\section{INTRODUCTION}

Idiopathic inflammatory myopathies (IIMs), including dermatomyositis (DM), polymyositis (PM) and inclusion body myositis (IBM), are a heterogeneous group of diseases

\section{Key messages}

What is already known about this subject?

- Although several studies have reported the characteristic muscle MRI findings in patients with idiopathic inflammatory myopathies (IIMs), no studies have conclusively revealed the distinctive characteristics that can differentiate dermatomyositis (DM) from other IIMs on MRI.

What does this study add?

- The characteristic MRI findings (short-tau inversion recovery [STIR] and gadolinium-enhanced fat-suppressed T1-weighted imaging [Gd-T1WI]) of skeletal muscles in patients with DM are high signal intensity (HSI) in subcutaneous adipose tissue and fasciae, peripheral distribution of HSI in muscle, and honeycomb pattern of $\mathrm{HSI}$ in muscle.

- The likelihood of DM score assessed by the characteristic MRI findings demonstrated good diagnostic performance in DM.

- MRI (STIR and Gd-T1WI) of myositis-specific autoantibodies or myositis-associated autoantibodies (MSAs/MAAs)-positive patients revealed more frequent fascial HSI but less frequent foggy pattern of HSI in muscle compared with MSAs/MAAs-negative patients.

How might this impact on clinical practice?

- The characteristic MRI findings of skeletal muscles could be of assistance in the diagnosis of patients with DM as well as the prediction of patients with MSAs/MAAs.

characterised by muscle weakness and inflammatory infiltrates in muscle tissue. ${ }^{1}$ These inflammatory myopathies have clinically and histopathologically different characteristics. Patients with IIMs can be classified by the 2017 European League Against Rheumatism/American College of Rheumatology (EULAR/ACR) classification criteria with 
high sensitivity and specificity, and further subclassified into the major subgroups. ${ }^{2}$

MRI of skeletal muscles is a useful tool for assessing disease activity in IIMs, evaluating accurate locations of lesions and identifying useful biopsy sites. ${ }^{3-7}$ Several studies have reported characteristic muscle MRI findings in patients with IIMs, including subcutaneous adipose tissue oedema, fascial oedema, patchy or diffuse distribution of muscle oedema, muscle atrophy and fatty replacement. ${ }^{8-14}$ For example, Cantwell et al $l^{8}$ noted that MRI showed subcutaneous tissue oedema in patients with DM, but not in patients with PM, and that muscle oedema was patchy in patients with DM, but diffuse in patients with PM. We previously reported that high signal intensity (HSI) in the fasciae on short-tau inversion recovery (STIR) or fat-suppressed T2-weighted images with enhancement on gadolinium-enhanced fat-suppressed T1-weighted imaging (Gd-T1WI), considered to reflect fascial oedema or inflammation, was more frequently detected in patients with DM than in patients with PM. ${ }^{9}$ Another study reported that the characteristic MRI findings in patients with IBM were fatty replacement of the flexor digitorum profundus, anterior thigh muscles with relative sparing of the rectus femoris and medial part of the gastrocnemius. ${ }^{12}$ Furthermore, a recent study reported a pattern of MRI findings in patients with IBM and its diagnostic accuracy in differentiating IBM from other myopathies, such as DM and PM. ${ }^{13}$ Although it is obvious from the above descriptions that individual IIMs are associated with different muscle MRI findings, no studies have conclusively revealed the distinctive characteristics that can differentiate DM from other IIMs on MRI. To address this issue, we analysed the various patterns of muscle MRI findings in patients with suspicion of IIMs, and compared each IIM with other myositis groups. The objective of our study was to define the characteristic findings on MRI of skeletal muscles in patients with DM relative to those in patients with other IIMs and to assess their diagnostic performance in DM.

\section{METHODS}

Patients

We retrospectively reviewed a cohort of adult patients who were admitted to the Division of Rheumatology (The Jikei University Hospital, Tokyo, Japan) between April 2007 and March 2016 with suspicion of IIMs. Using the 2017 EULAR/ ACR classification criteria, ${ }^{2}$ patients with definite (probability of $\geq 90 \%$ or total aggregate score of $\geq 7.5$ without muscle biopsy and $\geq 8.7$ with muscle biopsy) or probable (probability of $\geq 55 \%$ and $<90 \%$ or total aggregate score of $\geq 5.5$ and $<7.5$ without muscle biopsy and $\geq 6.7$ and $<8.7$ with muscle biopsy) IIM were classified as having IIM, and subsequently subclassified into the major subgroups by applying the classification tree. Meanwhile, patients with possible IIM (probability of $\geq 50 \%$ and $<55 \%$ or total aggregate score of $\geq 5.3$ and $<5.5$ without muscle biopsy and $\geq 6.5$ and $<6.7$ with muscle biopsy) or non-IIM (probability of $<50 \%$ or total aggregate score of $<5.3$ without muscle biopsy and $<6.5$ with muscle biopsy) by the EULAR/ACR criteria were classified as non-IIM. The study population comprised 88 patients who underwent MRI examinations of their proximal limb skeletal muscles. The following patient data were recorded: age; sex; skin rash; muscle weakness (manual muscle test score $<5$ ); myalgia; serum creatine kinase $(\mathrm{CK})$ level (normal range: 25-160 IU/L) on admission; presence of myositis-specific autoantibodies (MSAs) or myositis-associated autoantibodies (MAAs); skeletal muscle MRI findings; muscle biopsy findings; and time from onset of symptoms to first MRI. The MSAs included antibodies against aminoacyl-tRNA synthetases (ARS) (Jo-1, PL-7, PL-12, EJ, OJ), signal recognition particle (SRP), melanoma differentiation-associated gene 5 (MDA5), transcriptional intermediary factor $1-\gamma($ TIF1- $\gamma$ ) and nuclear helicase Mi-2. The MAAs included antibodies against PM-Scl 75, PM-Scl 100, $\mathrm{Ku}, \mathrm{Ro52}$ and U1-RNP. Sera were taken within 1 month of the MRI performance. The anti-U1-RNP antibodies were measured with a commercially available chemiluminescent enzyme immunoassay kit (STACIA MEBLux test RNP; Medical \& Biological Laboratories, Nagoya, Japan). The anti-TIF1 $-\gamma$ antibodies were measured with a commercially available ELISA kit (MESACUP anti-TIF1- $\gamma$ test; Medical \& Biological Laboratories). The anti-MDA5 antibodies were measured by an in-house ELISA using recombinant MDA5 as an antigen source. ${ }^{15}$ Other MSAs and MAAs (including anti-Jo-1, anti-PL-7, anti-PL-12, anti-EJ, anti-OJ, anti-SRP, antiMi-2, anti-PM-Scl 75, anti-PM-Scl 100, anti-Ku and anti-Ro52 antibodies) were detected by a commercially available line immunoassay (Euroline Myositis Profile 3; EUROIMMUN, Luebeck, Germany). The anti-MDA5 antibodies were measured at Keio University and the other MSAs and MAAs were measured at SRL (Tokyo, Japan). The study protocol was approved by the Ethics Committee of The Jikei University School of Medicine (Approval Number: 28-049 [8292]), and the requirement for informed consent was waived.

\section{Imaging}

Muscles in the upper arms and/or thighs were evaluated by MRI using a $1.5 \mathrm{~T}$ unit (MAGNETOM Symphony or Avanto; Siemens Healthcare, Erlangen, Germany). STIR imaging (repetition time: $4000 \mathrm{~ms}$; echo time: $25 \mathrm{~ms}$; inversion time: $180 \mathrm{~ms}$; slice thickness: $5.0 \mathrm{~mm}$; flip angle: $150^{\circ}$; field of view: $260 \times 260 \mathrm{~mm}$ in upper arms, $370 \times 370$ $\mathrm{mm}$ in thighs; matrix: $256 \times 256$ in upper arms, $345 \times 384$ in thighs; acquisition time: $153 \mathrm{~s}$ ) and Gd-T1WI (repetition time: $530 \mathrm{~ms}$; echo time: $11 \mathrm{~ms}$; inversion time: $5.0 \mathrm{~ms}$; flip angle: $150^{\circ}$; field of view: $335 \times 370 \mathrm{~mm}$; matrix: $326 \times 384$; acquisition time: $161 \mathrm{~s}$ ) were performed in the axial plane. Contrast media, gadopentetate dimeglumine (Magnevist; Bayer Yakuhin, Osaka, Japan), gadodiamide (Omniscan; Daiichi Sankyo, Tokyo, Japan) or gadoteridol (ProHance; Eisai, Tokyo, Japan) were administered at a dose of 0.2 $\mathrm{mmol} / \mathrm{kg}$ body weight.

\section{Evaluation of images}

One radiologist (with 15 years of experience in MRI interpretation) and one rheumatologist (with 21 years of clinical 


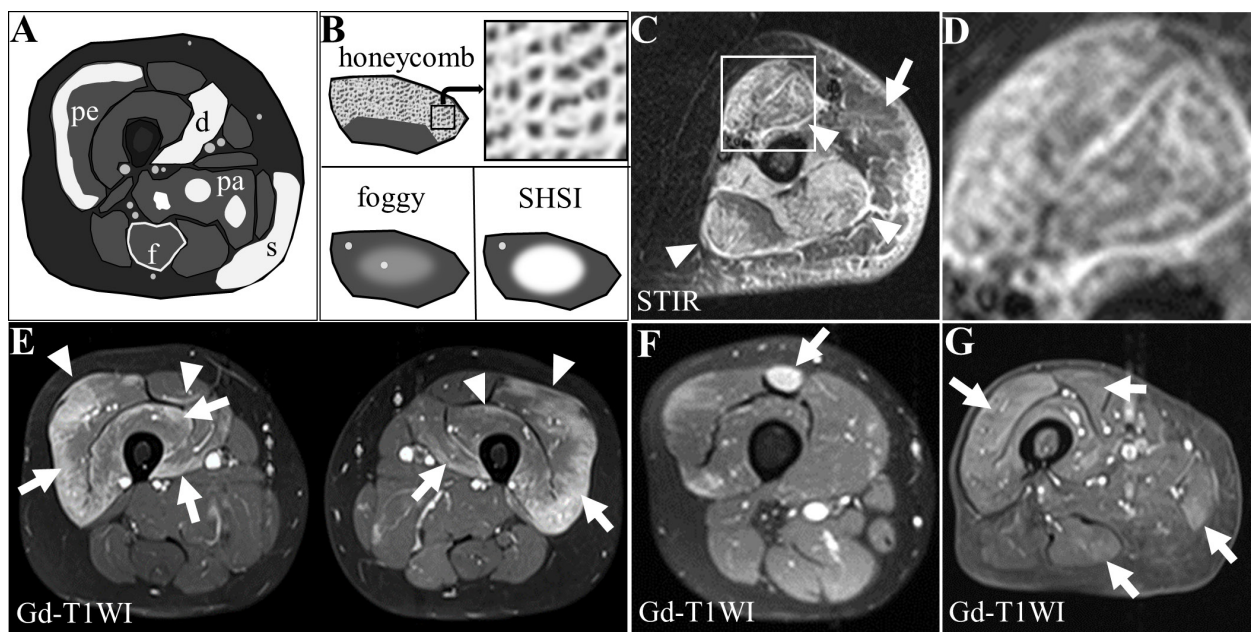

Figure 1 Examples of MRI findings in patients with DM or PM. (A) Structures with HSI (s, subcutaneous adipose tissue; $f$, fascia) and distributions of HSI areas in muscle (d, diffuse; pa, patchy; pe, peripheral) appear white. (B) Patterns of HSI in muscle. (C) STIR image of the left upper arm of a patient with DM. Structures with HSI are the subcutaneous adipose tissue (arrow), fasciae (arrowheads) and muscles. Distribution and pattern of HSI in the biceps muscle are diffuse and honeycomb, respectively (within the square). (D) Higher magnification image of the square in (C). (E) Gd-T1WI image of the thighs of a patient with DM. Structures with HSI are the bilateral quadriceps fasciae (arrowheads) and muscles. HSI distribution in the quadriceps muscles is peripheral (arrows). (F) Gd-T1WI image of the right thigh of a patient with DM. Distribution and pattern of $\mathrm{HSI}$ in the right rectus femoris muscle are diffuse and SHSI (arrow), respectively. (G) Gd-T1WI image of the right thigh of a patient with PM. There is diffuse HSI in the vastus lateralis muscle and patchy HSI in the rectus femoris, gracilis and semitendinosus muscles, producing a foggy pattern (arrows). DM, dermatomyositis; Gd-T1WI, gadolinium-enhanced fatsuppressed T1-weighted imaging; HSI, high signal intensity; PM, polymyositis; SHSI, strong high signal intensity; STIR, shorttau inversion recovery.

experience in rheumatology) interpreted the images separately. Any disagreements between the examiners were resolved by consensus with a second radiologist who had 21 years of experience in MRI interpretation. All examiners were blinded to the clinical information. The presence or absence of the following features in the axial plane was recorded: structures with HSI (subcutaneous adipose tissue, fascia, muscle); distributions of HSI areas in muscle (diffuse, patchy, peripheral) and patterns of HSI in muscle (honeycomb, foggy, strong high signal intensity [SHSI]). The presence of HSI on fasciae caused by chemical shift artefacts was excluded.

Figure 1A shows the structures with HSI and distributions of HSI areas in muscle. The distributions were described as 'diffuse', 'patchy' and/or 'peripheral'. 'Diffuse' distribution was used to describe HSI in the entire area of the involved muscle. 'Patchy' distribution was used when HSI areas were present discretely within a background of normal muscle signal intensity. 'Peripheral' distribution was used when HSI areas were present in the marginal zone of the involved muscle. Figure $1 \mathrm{~B}$ shows the patterns of HSI areas in muscle. The patterns were described as 'honeycomb', 'foggy' and/or 'SHSI'. 'Honeycomb' pattern was defined as a heterogeneous reticular pattern of the HSI area within muscle. 'Foggy' pattern was defined as a homogeneous faint HSI area in which the background of the vessel signal could be detected. 'SHSI' pattern was defined as a strong HSI area in which the background of the vessel signal could not be detected or an area of signal intensity that was higher than the highest signal intensity of the nearby vessels. If the 'foggy' pattern was seen contiguous with the 'honeycomb' pattern or 'SHSI' pattern, we described it as 'honeycomb' pattern or 'SHSI' pattern, respectively. Examples of actual MR images of patients with DM or PM are shown in figure $1 \mathrm{C}-\mathrm{G}$. Figure $1 \mathrm{C}$ shows the STIR image of the left upper arm of a patient with DM. In this image, the structures with HSI are the subcutaneous adipose tissue (arrow), fasciae (arrowheads), and biceps and triceps muscles. The distribution of the HSI area in the biceps is 'diffuse' (enclosed in a square). Figure 1D shows the higher magnification image of the square in figure 1C. The HSI in the biceps muscle has a 'honeycomb' pattern. The Gd-T1WI findings were in line with the STIR results. Figure 1E shows the Gd-T1WI image of the thighs of a patient with DM. Here, the structures with HSI are the bilateral quadriceps fasciae (arrowheads) and muscles (arrow). The distributions of the HSI areas in the bilateral vastus lateralis, bilateral vastus medialis and right vastus intermedius muscles are 'peripheral' (arrows). Figure 1F shows the Gd-T1WI image of the right thigh of a patient with DM. Here, the distribution and pattern of the HSI in the right rectus femoris muscle are 'diffuse' and 'SHSI' (arrow), respectively. Figure $1 \mathrm{G}$ shows the Gd-T1WI image of the right thigh of a patient with PM. HSI exhibits a 'diffuse' distribution in the vastus lateralis muscle and a 'patchy' distribution in the rectus femoris, gracilis and semitendinosus muscles, producing a 'foggy' pattern (arrows). The findings on the STIR images shown in figure $1 \mathrm{E}-\mathrm{G}$ are in line with the Gd-T1WI results. 


\section{Statistical analysis}

Fisher's exact test with the Benjamini-Hochberg correction $^{16}$ for multiple testing was used for comparisons of frequencies. The Kruskal-Wallis test with the Dwass, Steel, Critchlow-Fligner multiple pairwise comparison procedure was used for comparisons of median values. Cohen's kappa statistics and 95\% CIs were calculated to assess interobserver agreement in the interpretation of muscle MRI findings between the two examiners. According to the standards proposed by Landis and Koch, ${ }^{17}$ the strength of agreement for the kappa statistics was interpreted as follows: $<0.00$, poor; $0.00-0.20$, slight; $0.21-0.40$, fair; 0.41-0.60, moderate; $0.61-0.80$, substantial and 0.81-1.00, almost perfect. A binary logistic regression analysis with forward selection ( $p$ for entry=0.1) was performed to investigate whether variables related to patient characteristics were associated with the presence or absence of each MRI finding. Explanatory variables included age, sex, muscle weakness, myalgia, CK level, time from onset of symptoms to first MRI and diagnosis (DM, amyopathic DM (ADM), PM, non-IIM [reference variable]). A receiver-operating characteristic (ROC) analysis was performed to evaluate the diagnostic performance of characteristic MRI findings in diagnosing DM. For the ROC analysis, the likelihood of DM was scored by counting the number of significant characteristic MRI findings in patients with DM. The difference in diagnostic performance between STIR and Gd-T1WI was analysed by comparing the areas under the ROC curves (AUCs) according to the method described by DeLong et $a .^{18}$ The AUCs were internally validated using a cross-validation (jack-knife) method to correct the AUCs for optimism.

All statistical analyses were performed using SAS software (V.9.4 for Windows; SAS Institute, Cary, NC, USA). Values of $\mathrm{p}<0.05$ were considered to indicate statistical significance.

\section{RESULTS}

Patients

Of the 88 patients evaluated, 72 were classified as having IIM (43 with definite IIM and 29 with probable IIM) by the EULAR/ACR criteria. Among the 72 patients with IIM, 36 were classified as DM, 17 as ADM and 19 as PM. There were no patients with IBM in our study cohort. The remaining 16 patients were classified as non-IIM (one with possible IIM and 15 with non-IIM) by the EULAR/ACR criteria. Regarding the 16 patients with non-IIM, 4 were clinically diagnosed with anti-ARS antibody-positive myositis, 4 with definite or probable PM by the Bohan and Peter criteria, ${ }^{19} 3$ with anti-mitochondrial antibody-positive myositis, ${ }^{20} 2$ with systemic lupus erythematosus, 2 with systemic sclerosis and 1 with mixed connective tissue disease. Among the 36 patients with DM, 17 with ADM, 19 with PM and 16 with non-IIM, 33, 16, 16 and 15 patients were not taking any medications, respectively. The remaining patients were under treatment with corticosteroids, azathioprine, cyclosporine or methotrexate. Among the 8 patients under treatment, 3 patients with DM, 1 patient with ADM, 2 patients with
PM and 1 patient with non-IIM underwent MRI because of disease relapse during the tapering of corticosteroids, and the remaining one patient with DM underwent MRI at just 1 week after the initial treatment. The clinical characteristics of the patients in the IIM subgroups and the non-IIM group are shown in table 1.

\section{Differences in characteristic MRI findings among the groups}

STIR imaging was carried out for all 88 patients (36 with DM, 17 with ADM, 19 with PM and 16 with non-IIM), whereas Gd-T1WI was performed in 79 patients (32 with DM, 16 with ADM, 17 with PM and 14 with non-IIM). Interobserver agreement in the interpretation of muscle MRI findings between the two examiners was substantial to almost perfect (kappa 0.66 to 0.98 ; table 2 ).

Figure 2 shows the differences in percentage appearances of MRI findings among the patients in the IIM subgroups and the non-IIM group. The percentage appearances on both STIR and Gd-T1WI are as follows: subcutaneous HSI was significantly higher in the DM group compared with the other groups; fascial HSI was significantly higher in the DM and ADM groups compared with the PM and non-IIM groups; peripheral distribution was significantly higher in the DM group compared with the other groups; honeycomb pattern was significantly higher in the DM group compared with the ADM and PM groups; and foggy pattern was significantly lower in the DM group compared with the PM group. The percentage appearances of honeycomb pattern showed a significant difference between the DM and non-IIM groups on Gd-T1WI images only. The percentage appearances of foggy pattern showed a significant difference between the DM and non-IIM groups on STIR images only. The remaining findings (diffuse distribution, patchy distribution and SHSI pattern) showed no significant differences among the groups. SHSI pattern was only observed in untreated patients, and not in patients under treatment, while the remaining MRI findings were also observed in patients under treatment.

Binary logistic regression analysis (online supplementary figure S1) showed the following: (1) all defined MRI findings were not significantly associated with sex, muscle weakness and time from onset of symptoms to first MRI; (2) age was significantly associated with diffuse distribution (STIR and Gd-T1WI) and absence of patchy distribution (STIR); (3) myalgia was significantly associated with fascial HSI (STIR and Gd-T1WI) and peripheral distribution (STIR and Gd-T1WI), and absence of patchy distribution (Gd-T1WI); (4) CK level was significantly associated with diffuse distribution (STIR and Gd-T1WI), honeycomb pattern (STIR and Gd-T1WI) and foggy pattern (STIR and Gd-T1WI); (5) diagnosis of DM was significantly associated with subcutaneous HSI (STIR and Gd-T1WI), fascial HSI (STIR and Gd-T1WI), peripheral distribution (STIR and Gd-T1WI), and honeycomb pattern (STIR and Gd-T1WI), and absence of foggy pattern (STIR and Gd-T1WI); and (6) diagnosis of 
Table 1 Clinical characteristics of the patients in the IIM subgroups and the non-IIM group

\begin{tabular}{|c|c|c|c|c|c|}
\hline & $\begin{array}{l}\mathrm{DM} \\
(\mathrm{n}=36)\end{array}$ & $\begin{array}{l}\text { ADM } \\
(n=17)\end{array}$ & $\begin{array}{l}P M \\
(n=19)\end{array}$ & $\begin{array}{l}\text { Non-IIM* } \\
(n=16)\end{array}$ & $p<0.05$ \\
\hline Age (years), mean (SD) & $53.5(14.3)$ & $50.5(11.7)$ & $64.1(11.4)$ & $54.6(15.8)$ & $\begin{array}{l}\text { DM versus } P M \\
A D M \text { versus PM }\end{array}$ \\
\hline Female, n (\%) & $24(66.7)$ & $8(47.1)$ & $12(63.2)$ & $11(68.8)$ & NSD \\
\hline Muscle weakness, n (\%) & $34(94.4)$ & $2(11.8)$ & $15(79.0)$ & $10(62.5)$ & $\begin{array}{l}\text { DM versus ADM } \\
\text { DM versus non- } \\
\text { IIM } \\
\text { ADM versus PM } \\
\text { ADM versus non- } \\
\text { IIM }\end{array}$ \\
\hline Myalgia, n (\%) & $24(66.7)$ & $11(64.7)$ & $8(42.1)$ & $8(50.0)$ & NSD \\
\hline CK level (IU/litre), mean (SD) & $1771.9(2557.8)$ & $394.6(586.9)$ & $1840.1(1651.4)$ & $1423.1(1456.7)$ & $\begin{array}{l}\mathrm{DM} \text { versus } \mathrm{ADM} \\
\mathrm{ADM} \text { versus } \mathrm{PM} \\
\mathrm{ADM} \text { versus non- } \\
\mathrm{IIM}\end{array}$ \\
\hline $\begin{array}{l}\text { Time from onset of symptoms to first MRI } \\
\text { (days), mean (SD) }\end{array}$ & $157.9(243.6)$ & $205.4(394.6)$ & $495.6(622.0)$ & $454.4(711.5)$ & DM versus $\mathrm{PM}$ \\
\hline Muscle biopsy performed, n (\%) & $29(80.6)$ & $10(58.8)$ & $15(78.9)$ & $9(86.7) \dagger$ & NSD \\
\hline Inflammatory cell infiltration in muscle, $n(\%)$ & 25 (86.2) & $7(70.0)$ & $15(100.0)$ & $9(100.0)$ & NSD \\
\hline
\end{tabular}

*Non-IIM included anti-ARS antibody-positive myositis $(n=4)$, definite or probable PM by the Bohan and Peter criteria ( $n=4)$, antimitochondrial antibody-positive myositis $(n=3)$, SLE $(n=2)$, SSc $(n=2)$ and MCTD $(n=1)$.

†Muscle biopsy was performed in nine patients with non-IIM: anti-ARS antibody-positive myositis $(n=2)$, definite or probable PM by the Bohan and Peter criteria ( $n=3)$, anti-mitochondrial antibody-positive myositis $(n=2)$, SLE $(n=1)$, SSc $(n=1)$.

ADM, amyopathic dermatomyositis; ARS, aminoacyl-tRNA synthetases; CK, creatine kinase; DM, dermatomyositis;IIM, idiopathic inflammatory myopathy; MCTD, mixed connective tissue disease; NSD, no significant differences among the subgroups; PM, polymyositis; SLE, systemic lupus erythematosus; SSc, systemic sclerosis.

ADM was significantly associated with fascial HSI (STIR and Gd-T1WI).

Thus, even considering the intergroup imbalances in the patient characteristics (table 1), the significant characteristic MRI findings in patients with DM on both STIR and Gd-T1WI were subcutaneous HSI, peripheral distribution in muscle and honeycomb pattern in muscle. Fascial HSI was considered a characteristic MRI finding in not only patients with DM but also patients with ADM.
Differences in characteristic MRI findings between the MSAs/ MAAs-positive and MSAs/MAAs-negative groups

We divided 77 of our patients into an MSAs/MAAs-positive group (55 patients with MSAs and/or MAAs) and an MSAs/MAAs-negative group (22 patients without MSAs/ MAAs). The remaining 11 patients had insufficient data for these autoantibodies and were excluded. Of the 55 patients with MSAs/MAAs, 19 had anti-ARS antibodies, 5 had anti-SRP antibodies, 12 had anti-MDA5 antibodies,

Table 2 Interobserver agreement in the interpretation of muscle MRI findings

\begin{tabular}{l} 
Kappa statistics, $95 \% \mathrm{CI}$ \\
\hline STIR ( $=88)$
\end{tabular}

\begin{tabular}{|c|c|c|}
\hline \multicolumn{3}{|l|}{ MRI findings } \\
\hline Subcutaneous HSI & 0.98 (0.93 to 1.00$)$ & 0.97 (0.93 to 1.00$)$ \\
\hline Fascial HSI & 0.66 (0.50 to 0.82$)$ & 0.72 (0.57 to 0.87$)$ \\
\hline Diffuse distribution & $0.70(0.55$ to 0.86$)$ & 0.81 (0.67 to 0.94$)$ \\
\hline Patchy distribution & $0.72(0.56$ to 0.89$)$ & 0.71 (0.54 to 0.89$)$ \\
\hline Foggy pattern & 0.79 (0.62 to 0.97$)$ & $0.86(0.70$ to 1.00$)$ \\
\hline SHSI pattern & $0.82(0.63$ to 1.00$)$ & $0.72(0.47$ to 0.98$)$ \\
\hline
\end{tabular}

STIR, short-tau inversion recovery; Gd-T1WI, gadolinium-enhanced fat-suppressed T1-weighted imaging; HSI, high signal intensity; SHSI, strong high signal intensity. 


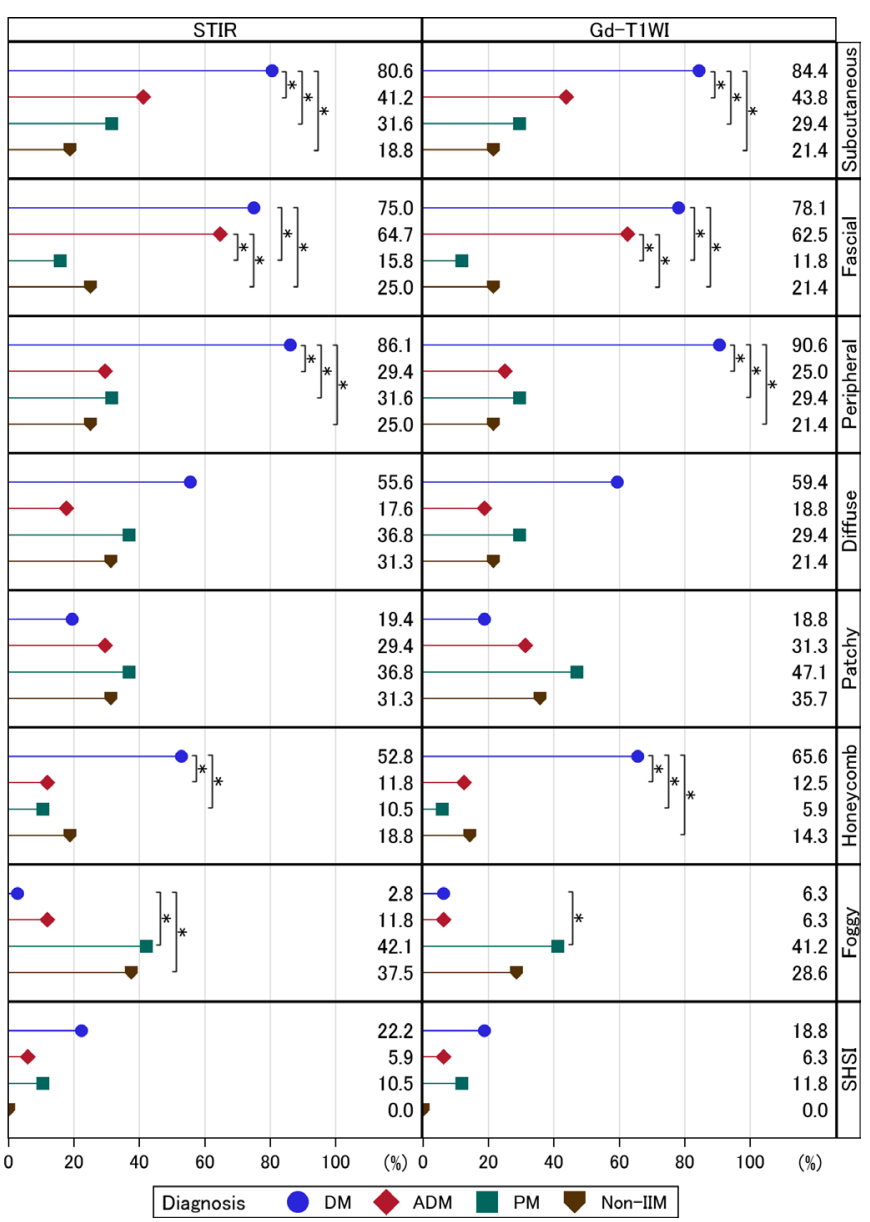

Figure 2 Percentage appearances of MRI findings among the patients in the IIM subgroups and the non-IIM group. The percentage appearances of subcutaneous HSI, fascial $\mathrm{HSI}$, peripheral/diffuse/patchy distributions of $\mathrm{HSI}$ in muscle, and honeycomb/foggy/SHSI patterns of HSI in muscle were analysed among the patients in the IIM subgroups and the non-IIM group. STIR and Gd-T1WI images were obtained. * $\mathrm{p}<0.05$. ADM, amyopathic dermatomyositis; DM, dermatomyositis; Gd-T1WI, gadolinium-enhanced fat-suppressed T1-weighted imaging; IIM, idiopathic inflammatory myopathy; HSI, high signal intensity; PM, polymyositis; SHSI, strong high signal intensity; STIR, shorttau inversion recovery.

2 had anti-TIF1- $\gamma$ antibodies, 7 had anti-Mi-2 antibodies, 1 had anti- PM-Scl 75 antibodies, 3 had anti-Ku antibodies and 6 had anti-U1-RNP antibodies. Anti-Ro52 antibodies were simultaneously detected in four patients with anti-ARS antibodies, one patient with anti-SRP antibodies, three patients with anti-MDA5 antibodies and one patient with anti-Ku antibodies.

Figure 3 shows the differences in percentage appearances of MRI findings between the MSAs/MAAs-positive and MSAs / MAAs-negative groups. On STIR and Gd-T1WI, the percentage appearances of fascial HSI were significantly higher in the MSAs/MAAs-positive group than in the MSAs/MAAs-negative group. Among the 19 patients with anti-ARS antibodies, 5 with anti-SRP antibodies, 12 with anti-MDA 5 antibodies, 2 with anti-TIF $1-\gamma$ antibodies,
7 with anti-Mi-2 antibodies, 1 with anti-PM-Scl 75 antibodies, 3 with anti-Ku antibodies and 6 with anti-U1-RNP antibodies, 12, 0, 7, 2, 7, 0, 2 and 3 patients had fascial HSI detected on STIR images, respectively. Fascial HSI on MRI was observed in all of the MSAs/MAAs-positive PM patients, but none of the MSAs/MAAs-negative PM patients. Among the MSAs/MAAs-positive PM patients with fascial HSI on MRI, two patients had anti-ARS antibodies and one patient had anti-Ku antibodies. Foggy pattern was significantly more frequently observed in the MSAs/MAAs-negative group compared with the MSAs/ MAAs-positive group. The remaining findings (subcutaneous HSI, peripheral distribution, diffuse distribution, patchy distribution, honeycomb pattern and SHSI pattern) showed no significant differences between the two groups.

\section{Diagnostic performance of characteristic MRI findings in DM}

The likelihood of DM was scored from 0 to 4 according to the number of the following characteristic MRI findings: subcutaneous HSI, fascial HSI, peripheral distribution and honeycomb pattern. As shown in figure 4, ROC analysis showed that the optimal cut-off point for the likelihood of DM score was $\geq 3$ (STIR: sensitivity $72.2 \%$, specificity $88.5 \%$, AUC $84.9 \%$, optimism-corrected AUC $76.7 \%$; Gd-T1WI: sensitivity $81.2 \%$, specificity $91.5 \%$, AUC 89.9\%, optimism-corrected AUC 81.3\%). In 79 patients who underwent both STIR and Gd-T1WI, there was no significant difference between the AUCs for STIR and Gd-T1WI (STIR: AUC 88.4\%; Gd-T1WI: AUC 89.9\%; $\mathrm{p}=0.12$ ). There were 6 patients who were not classified as DM by the EULAR/ACR criteria despite having likelihood of DM scores $\geq 3$, and all 6 patients were positive for MSAs/MAAs (1 ADM with anti-U1-RNP antibodies, 2 PM with anti-Ku antibodies, 1 non-IIM with anti-U1-RNP antibodies and 2 non-IIM with anti-ARS antibodies).

\section{DISCUSSION}

We found potentially distinctive characteristics for skeletal muscle MRI findings in patients with DM. In patients with DM, HSI was predominantly observed in the subcutaneous adipose tissue, fasciae and peripheral areas of muscle, and the HSI in muscle showed a honeycomb pattern rather than a foggy pattern.

Cantwell $e t a l^{8}$ reported that STIR showed HSI in subcutaneous tissue in patients with DM while no significant HSI was detected in patients with PM. Similar to their report, our study revealed that subcutaneous HSI on both STIR and Gd-T1WI was predominantly detected in patients with DM. Their report and the present data suggest that subcutaneous HSI on MRI is frequently present in patients with DM.

In our study, MRI revealed frequent HSI in the fasciae of patients with DM and ADM. Kimball et $a l^{11}$ reported that fascial oedema or inflammation on STIR was common in juvenile DM. Allen $e t a l^{21}$ and Tsuruta $e t a l^{22}$ reported cases of adult-onset ADM with fasciitis detected by MRI 


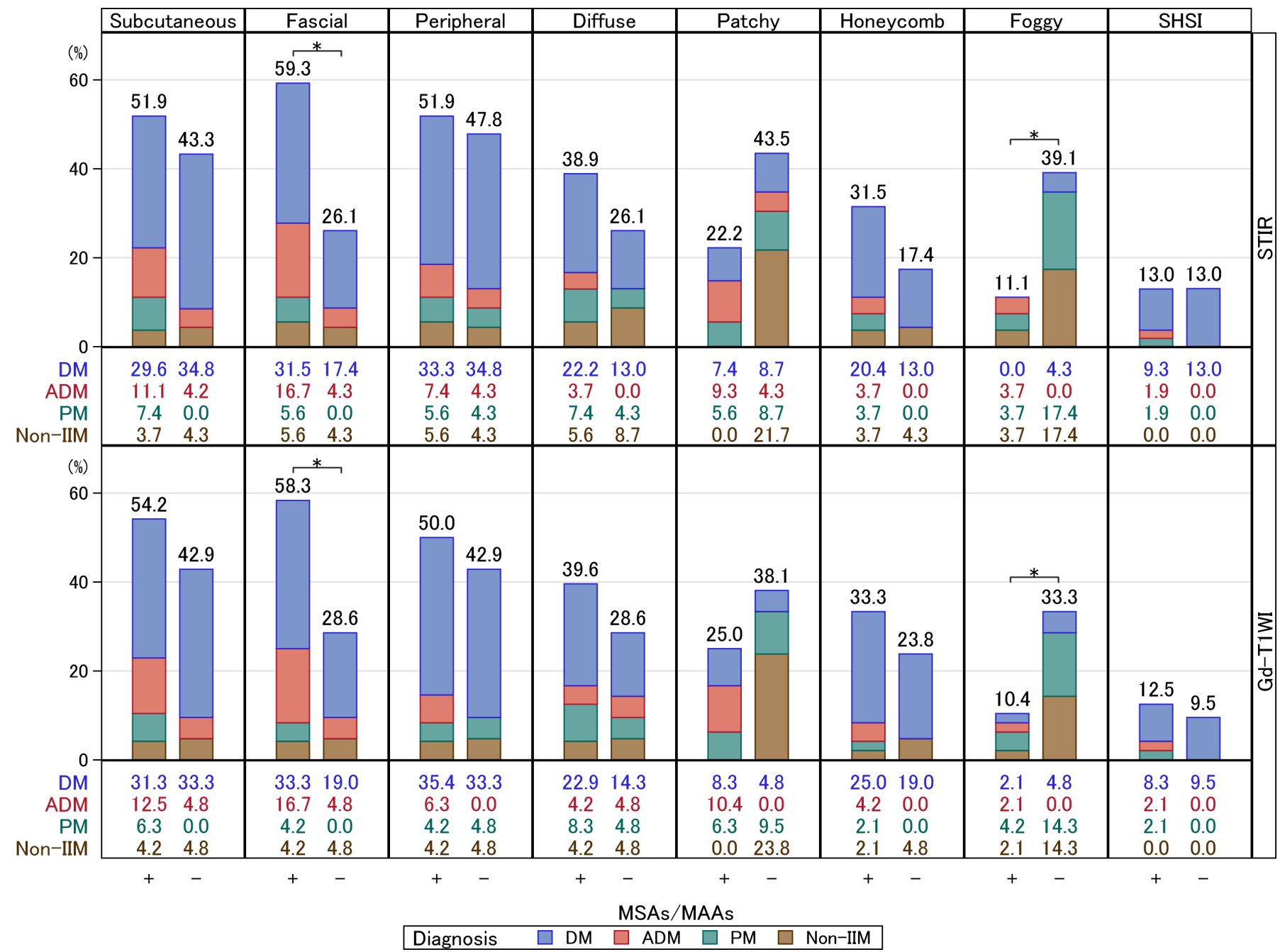

Figure 3 Percentage appearances of MRI findings in the MSAs/MAAs-positive and MSAs/MAAs-negative groups.

The percentage appearances of subcutaneous $\mathrm{HSI}$, fascial HSI, peripheral/diffuse/patchy distribution of $\mathrm{HSI}$ in muscle and honeycomb/foggy/SHSI patterns of HSI in muscle were compared between the MSAs/MAAs-positive and MSAs/ MAAs-negative groups. STIR and Gd-T1WI images were evaluated. ${ }^{*} p<0.05$. ADM, amyopathic dermatomyositis; DM, dermatomyositis; Gd-T1WI, gadolinium-enhanced fat-suppressed T1-weighted imaging; HSI, high signal intensity; IIM, idiopathic inflammatory myopathy; MAAs, myositis-associated autoantibodies; MSAs, myositis-specific autoantibodies; PM, polymyositis; SHSI, strong high signal intensity; STIR, short-tau inversion recovery.

with pathological correlations. In contrast, we previously showed that histopathologically evident fasciitis existed in not only adult-onset ADM but also adult-onset DM. ${ }^{9}$ We further demonstrated by MRI, power Doppler ultrasonography and en bloc biopsy that increased vascularity was present in the fasciae of patients with DM, but not patients with PM. ${ }^{23}{ }^{24}$ Consistent with our previous histopathological analyses, the present study showed that fascial HSI was more frequently detected in patients with DM than in patients with PM. Furthermore, we recently reported that myalgia in patients with DM or PM was associated with fasciitis, rather than myositis. ${ }^{25}$ In this study, fascial HSI and peripheral distribution of HSI in muscle were significantly associated with myalgia. Therefore, we speculated that not only fasciitis but also myositis distributed in the marginal zone of muscles may be related to myalgia.
With regard to the HSI distributions in muscle on MRI, we demonstrated that the peripheral distribution was characteristic of DM while the diffuse and patchy distributions showed no significant differences among the groups. Our findings differed from those of Cantwell et $a l,{ }^{11}$ who found that HSI in muscle on STIR images was diffuse in patients with PM, but patchy in patients with DM. Their study limitations included a small number of patients (2 patients with DM and 5 patients with $\mathrm{PM}$ ) and a lack of statistical analysis. We previously provided evidence that inflammation progressed from the fascia to the muscle in patients with $\mathrm{DM} .{ }^{9}$ The present results and our previous findings suggest that, in patients with DM, inflammation of the fascia during the initial stage of the disease may appear as fascial HSI on MRI, and subsequently change to the peripheral distribution of HSI with progression of the inflammation. 
A

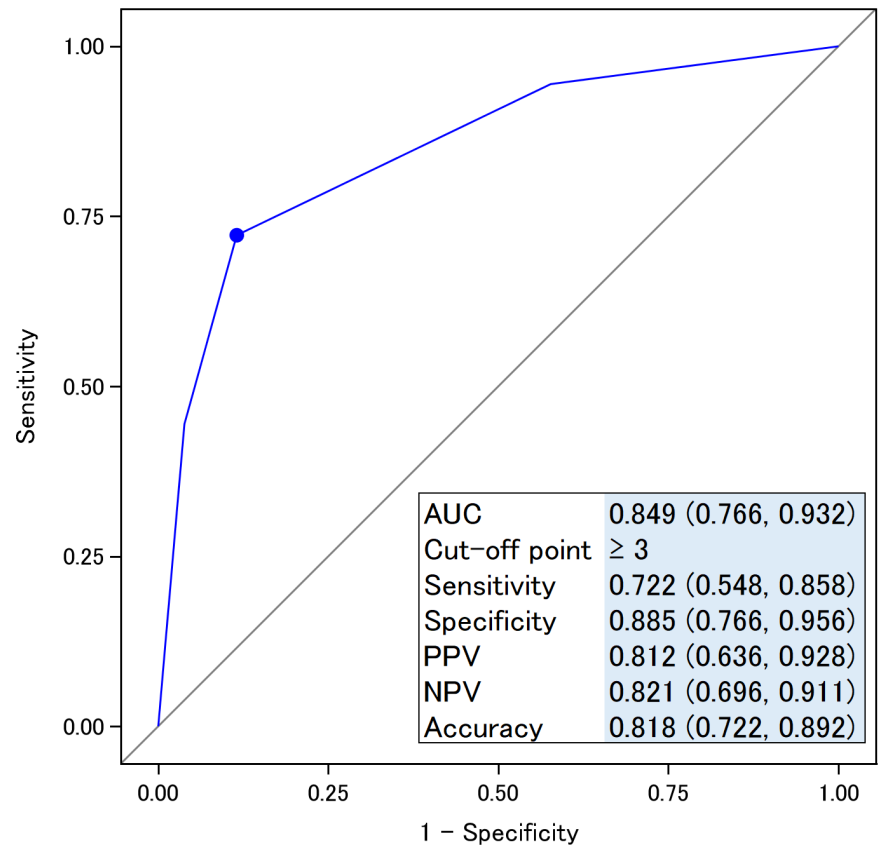

B

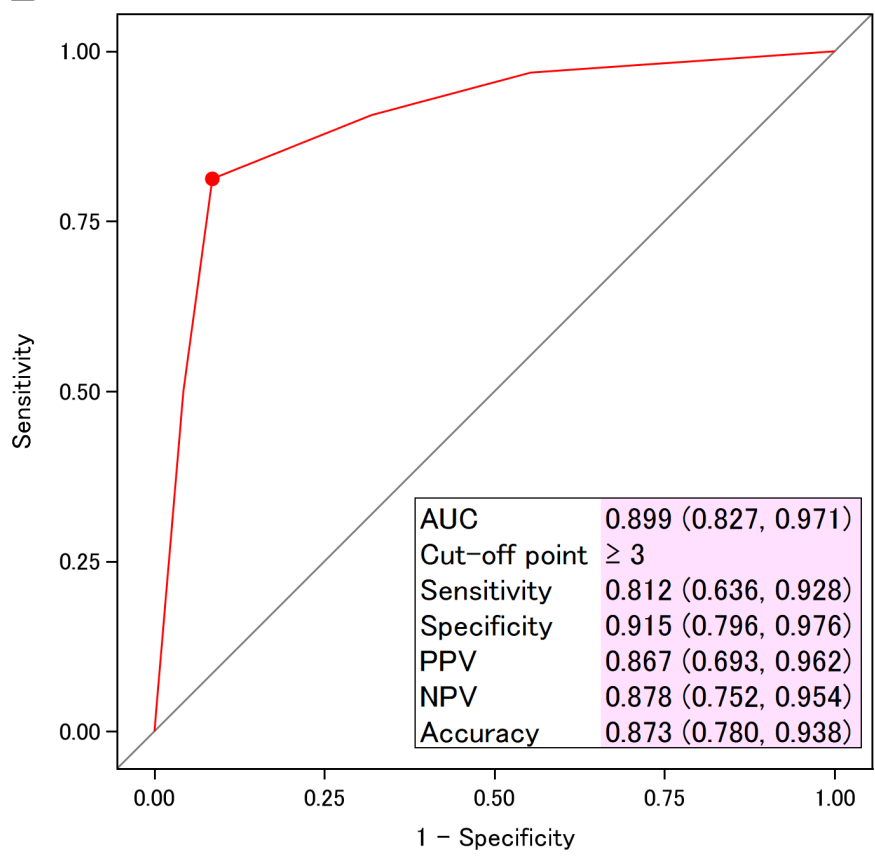

Figure 4 Diagnostic performance of characteristic muscle MRI findings in DM. The likelihood of DM was scored from 0 to 4 according to the number of significant characteristic MRI findings in patients with DM. ROC analysis showed that the optimal cut-off point for the likelihood of DM score was $\geq 3$. (A) STIR and (B) Gd-T1WI images were evaluated. AUC, area under ROC curve; DM, dermatomyositis; Gd-T1WI, gadolinium-enhanced fat-suppressed T1-weighted imaging; NPV, negative predictive value; PPV, positive predictive value; STIR, short-tau inversion recovery; ROC, receiver-operating characteristic.

Regarding the HSI patterns in muscle, the honeycomb pattern rather than the foggy pattern was frequently found in patients with DM. If a patient has the foggy pattern on MRI, a diagnosis of PM or non-IIM other than DM should be considered because it was particularly rare for patients with DM to exhibit the foggy pattern. Histopathologically, inflammatory cells predominantly infiltrate perivascular sites or interfascicular septa and surround the fascicles in DM. ${ }^{126-28}$ Further studies are needed to determine whether the pathological characteristics of DM reflect the honeycomb pattern of HSI in muscle observed on MRI.

Some previous studies reported that Gd-T1WI was not superior for assessment of inflammatory myopathies compared with conventional T1/T2-weighted spin-echo sequences. ${ }^{29-31}$ In our study, there was no significant difference in diagnostic performance between STIR and Gd-T1WI. Given the cost and the risk of complications associated with contrast media, achieving good diagnostic performance with STIR alone is beneficial to patients. However, Gd-T1WI showed superiority to STIR for detection of the honeycomb pattern because Gd-T1WI revealed significant differences between DM and all other IIM groups while STIR only showed significant differences between DM, ADM, and PM. Further studies are required to address whether assessment by STIR alone has sufficient diagnostic performance in IIMs.

We further found that MSAs/MAAs-positive patients more frequently showed fascial HSI on MRI than MSAs/ MAAs-negative patients while the foggy pattern was more frequent in MSAs/MAAs-negative patients than in MSAs/ MAAs-positive patients. This pattern of MRI findings in MSAs/MAAs-positive patients was partially similar to that in patients with DM. Andersson $e t a l^{32}$ reported that fascial oedema of thigh muscles on MRI was present in 19 of 66 $(29 \%)$ patients with anti-synthetase syndrome, and that 16 of the 19 patients with anti-synthetase syndrome had known myositis (8 patients with DM and 8 patients with PM). They speculated that the observed MRI pattern with concurrent oedema in muscle and fascia was a feature of active anti-synthetase syndrome, regardless of DM or PM. In the present study, 12 of 19 patients with anti-ARS antibodies, 7 of 12 with anti-MDA5 antibodies, 2 of 2 with anti-TIF1- $\gamma$ antibodies, 7 of 7 with anti-Mi-2 antibodies, 2 of 3 with anti-Ku antibodies and 3 of 6 with anti-U1-RNP antibodies had fascial HSI on STIR images. In addition, all of the patients with PM with fascial HSI on MRI were positive for MSAs/MAAs, such as anti-ARS antibodies and anti-Ku antibodies while none of the MSAs/MAAs-negative PM patients had fascial HSI on MRI. Thus, we speculated that not only anti-ARS antibodies but also other MSAs/MAAs were related to fascial HSI on MRI. However, the examined MSAs/MAAs were limited, and lacked some important autoantibodies that are currently in regular clinical use, such as anti-3-hydroxy-3-methylglutaryl-coenzyme A reductase or anti-small ubiquitin-like modifier activating enzyme (SAE) antibodies. Further studies are needed to assess the extent to which individual autoantibodies are associated with the presence of fascial HSI on MRI. 
To date, MRI has not been used as a variable in most classification or diagnostic criteria for IIMs because of its limited availability and undetermined specificity. ${ }^{33}$ However, we showed that MRI could be a highly specific modality for diagnosis of DM using our likelihood of DM score. Furthermore, and interestingly, all the false-positive cases for the likelihood of DM score on MRI were positive for MSAs/MAAs. Therefore, even if patients are not classified as DM according to the EULAR/ACR criteria, patients with likelihood of DM scores $\geq 3$ on MRI should be considered to possibly have MSAs/MAAs.

Our study has several limitations. First, we lacked normal control subjects and patients with IBM. However, in our limited study, the higher counts of DM pattern MRI findings increased the specificity of the diagnosis. Therefore, the characteristic MRI findings of skeletal muscle in patients with DM can be of assistance in the diagnosis of DM. Second, the study lacked quantitative evaluation of the MRI findings. To enhance reproducibility in the evaluation of our MRI findings, we evaluated the interobserver agreements for the individual MRI findings. Third, we did not perform an external validation study to confirm the accuracy of the diagnostic values in the study. Although our internal validation study with a cross-validation (jack-knife) method showed fair to good diagnostic performance (optimism-corrected AUC: STIR 76.7\%; Gd-T1WI 81.3\%), further external validation studies are needed to generalise our diagnostic methods. Fourth, the study had no contradistinctions between histopathological and MRI findings. Further studies are needed to confirm the concordance between these findings.

In conclusion, the likelihood of DM score assessed by the characteristic MRI findings demonstrated good diagnostic performance in DM. All the patients with false-negative results for the likelihood of DM score on muscle MRI were positive for MSAs/MAAs. In addition, the MRI findings for MSAs/MAAs-positive patients included more frequent fascial HSI, but less frequent foggy pattern of HSI in muscle compared with MSAs/MAAs-negative patients. Thus, the characteristic MRI findings of skeletal muscles could be of assistance in the diagnosis of DM as well as the prediction of patients with MSAs/ MAAs. However, the role of dedicated musculoskeletal MRI analysis, as described in our study, in the routine clinical management of patients with DM remains to be established.

\section{Author affiliations}

${ }^{1}$ Division of Rheumatology, Department of Internal Medicine, The Jikei University School of Medicine, Tokyo, Japan

${ }^{2}$ Department of Radiology, The Jikei University School of Medicine, Tokyo, Japan

Acknowledgements The authors thank Dr Masataka Kuwana (Department of Allergy and Rheumatology, Nippon Medical School Graduate School of Medicine, Tokyo, Japan) for his help in the detection of anti-MDA5 antibodies.

Contributors TU and KY contributed equally to the study. TU: study design, acquisition, analysis, and interpretation of data, drafting and critical revision of article. KY: study design, reading of MR images, acquisition and interpretation of data, drafting and critical revision of article. SM and GK: reading of MR images, interpretation of data, critical revision of article. $\mathrm{KN}$, KF and DK: data interpretation, critical revision of article. All authors read and approved the final article.

Funding The authors have not declared a specific grant for this research from any funding agency in the public, commercial or not-for-profit sectors.

Competing interests None declared.

Patient consent for publication Not required.

Ethics approval The study protocol was approved by the Ethics Committee of Jikei University School of Medicine (Approval Number: 28-049[8292]).

Provenance and peer review Not commissioned; externally peer reviewed. Data sharing statement No additional data are available

Open access This is an open access article distributed in accordance with the Creative Commons Attribution Non Commercial (CC BY-NC 4.0) license, which permits others to distribute, remix, adapt, build upon this work non-commercially, and license their derivative works on different terms, provided the original work is properly cited, appropriate credit is given, any changes made indicated, and the use is non-commercial. See: http://creativecommons.org/licenses/by-nc/4.0/.

\section{REFERENCES}

1. Dalakas MC. Polymyositis, dermatomyositis and inclusion-body myositis. N Engl J Med 1991;325:1487-98.

2. Lundberg IE, Tiärnlund $A$, Bottai M, et al. 2017 European League against Rheumatism/American College of rheumatology classification criteria for adult and juvenile idiopathic inflammatory myopathies and their major subgroups. Ann Rheum Dis 2017;76:1955-64.

3. Fraser DD, Frank JA, Dalakas MC. Inflammatory myopathies: MR imaging and spectroscopy. Radiology 1991;179:341-2.

4. Fraser DD, Frank JA, Dalakas M, et al. Magnetic resonance imaging in the idiopathic inflammatory myopathies. J Rheumatol 1991;18:1693-700.

5. Fujino $\mathrm{H}$, Kobayashi $\mathrm{T}$, Goto I, et al. Magnetic resonance imaging of the muscles in patients with polymyositis and dermatomyositis. Muscle Nerve 1991;14:716-20.

6. Hernandez RJ, Keim DR, Sullivan DB, et al. Magnetic resonance imaging appearance of the muscles in childhood dermatomyositis. $J$ Pediatr 1990;117:546-50.

7. Hernandez RJ, Keim DR, Chenevert TL, et al. Fat-suppressed MR imaging of myositis. Radiology 1992;182:217-9.

8. Cantwell C, Ryan M, O'Connell M, et al. A comparison of inflammatory myopathies at whole-body turbo stir MRI. Clin Radiol 2005;60:261-7.

9. Yoshida K, Kurosaka D, Joh K, et al. Fasciitis as a common lesion of dermatomyositis, demonstrated early after disease onset by en bloc biopsy combined with magnetic resonance imaging. Arthritis Rheum 2010;62:3751-9.

10. O'Connell MJ, Powell T, Brennan D, et al. Whole-body MR imaging in the diagnosis of polymyositis. AJR Am J Roentgenol 2002:179:967-71.

11. Kimball $A B$, Summers RM, Turner M, et al. Magnetic resonance imaging detection of occult skin and subcutaneous abnormalities in juvenile dermatomyositis. Implications for diagnosis and therapy. Arthritis Rheum 2000;43:1866-73.

12. Cox FM, Reijnierse M, van Rijswijk CSP, et al. Magnetic resonance imaging of skeletal muscles in sporadic inclusion body myositis. Rheumatology 2011;50:1153-61.

13. Tasca G, Monforte M, De Fino C, et al. Magnetic resonance imaging pattern recognition in sporadic inclusion-body myositis. Muscle Nerve 2015;52:956-62.

14. Pinal-Fernandez I, Casal-Dominguez M, Carrino JA, et al. Thigh muscle MRI in immune-mediated necrotising myopathy: extensive oedema, early muscle damage and role of anti-SRP autoantibodies as a marker of severity. Ann Rheum Dis 2017;76:681-7.

15. Sato S, Hoshino K, Satoh T, et al. RNA helicase encoded by melanoma differentiation-associated gene 5 is a major autoantigen in patients with clinically amyopathic dermatomyositis: association with rapidly progressive interstitial lung disease. Arthritis Rheum 2009:60:2193-200.

16. Benjamini Y, Hochberg Y. Controlling the false discovery rate: a practical and powerful approach to multiple testing. $J R$ Stat Soc $B$ 1995;57:289-300.

17. Landis JR, Koch GG. An application of hierarchical kappa-type statistics in the assessment of majority agreement among multiple observers. Biometrics 1977:33:363-74.

18. DeLong ER, DeLong DM, Clarke-Pearson DL. Comparing the areas under two or more correlated receiver operating characteristic curves: a nonparametric approach. Biometrics 1988;44:837-45. 
19. Bohan A, Peter JB. Polymyositis and dermatomyositis (second of two parts). N Engl J Med 1975;292:403-7.

20. Albayda J, Khan A, Casciola-Rosen L, et al. Inflammatory myopathy associated with anti-mitochondrial antibodies: a distinct phenotype with cardiac involvement. Semin Arthritis Rheum 2018;47:552-6.

21. Allen E, Schmahmann S, Sauser D, et al. Fasciitis in amyopathic dermatomyositis. J Clin Rheumatol 2003;9:51-3.

22. Tsuruta $\mathrm{Y}$, Ikezoe $\mathrm{K}$, Nakagaki $\mathrm{H}$, et al. A case of dermato-fasciitis: amyopathic dermatomyositis associated with fasciitis. Clin Rheumatol 2004;23:160-2.

23. Yoshida K, Nishioka M, Matsushima S, et al. Brief report: power Doppler ultrasonography for detection of increased vascularity in the fascia: a potential early diagnostic tool in fasciitis of dermatomyositis. Arthritis Rheumatol 2016;68:2986-91.

24. Yoshida K, Ito H, Furuya K, et al. Angiogenesis and VEGF-expressing cells are identified predominantly in the fascia rather than in the muscle during the early phase of dermatomyositis. Arthritis Res Ther 2017;19.

25. Noda K, Yoshida K, Ukichi T, et al. Myalgia in patients with dermatomyositis and polymyositis is attributable to fasciitis rather than myositis: a retrospective study of 32 patients who underwent histopathological examinations. J Rheumatol 2017;44:482-7.

26. Engel AG, Arahata K. Monoclonal antibody analysis of mononuclear cells in myopathies. II: Phenotypes of autoinvasive cells in polymyositis and inclusion body myositis. Ann Neurol 1984;16:209-15.

27. Mantegazza R, Bernasconi P. Cellular aspects of myositis. Curr Opin Rheumatol 1994;6:568-74.

28. Vattemi G, Mirabella M, Guglielmi V, et al. Muscle biopsy features of idiopathic inflammatory myopathies and differential diagnosis. Auto Immun Highlights 2014;5:77-85.

29. Stiglbauer R, Graninger W, Prayer L, et al. Polymyositis: MRIappearance at $1.5 \mathrm{~T}$ and correlation to clinical findings. Clin Radiol 1993;48:244-8

30. Reimers CD, Schedel H, Fleckenstein JL, et al. Magnetic resonance imaging of skeletal muscles in idiopathic inflammatory myopathies of adults. J Neurol 1994;241:306-14.

31. Schedel $\mathrm{H}$, Reimers CD, Vogl T, et al. Muscle edema in MR imaging of neuromuscular diseases. Acta Radiol 1995;36:228-32.

32. Andersson H, Kirkhus E, Garen T, et al. Comparative analyses of muscle MRI and muscular function in anti-synthetase syndrome patients and matched controls: a cross-sectional study. Arthritis Res Ther 2017;19.

33. Day J, Patel S, Limaye V. The role of magnetic resonance imaging techniques in evaluation and management of the idiopathic inflammatory myopathies. Semin Arthritis Rheum 2017;46:642-9. 